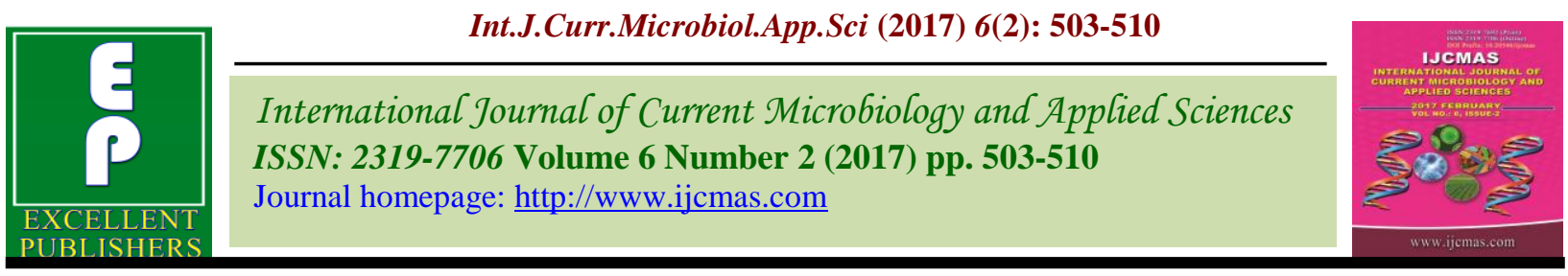

Original Research Article

http://dx.doi.org/10.20546/ijcmas.2017.602.057

\title{
Survey of Papaya Mealybug Paracoccus marginatus on Tapioca Crop in Different Districts of Tamil Nadu
}

\author{
K. Indirakumar*, J.S. Kennedy and M. Devi \\ Department of Agricultural Entomology, Tamil Nadu Agricultural University, \\ Coimbatore, Tamil Nadu, India \\ *Corresponding author
}

\begin{abstract}
A B S T R A C T
Keywords

Papaya mealybug, Tapioca, varieties, Incidence, Pest intensity, Different Districts.

Article Info

Accepted:

15 January 2017

Available Online:

10 February 2017

The extensive survey was made during year 2011 to 2012 in different blocks of Coimbatore, Erode, Perambalur, Salem and Tirupur districts of Tamil Nadu on the incidence of papaya mealybug, P. marginatus on tapioca. Among the ninety five farmer's holdings observed, none of the field was free from the pest incidence. The incidence ranged from 2 to 100 per cent. All the plant parts were covered with mealybugs with crinkling of leaves and leaves and fruits covered with honey dew and sooty mold and were started drying. Among the 95 fields surveyed, 13 recorded very high incidence, 3 with high incidence, 5 with medium incidence, 22 with low incidence and 52 with very low incidence. Very high incidence of 100 per cent was observed in only eight fields. Complete shedding of leaves occurred in most of the plants.
\end{abstract}

\section{Introduction}

Tapioca is affected by several arthropods, that can be considered as key or secondary pests including painted grasshopper (Poekilocerus pictus Fab.), aphids (Aphis gossypii Glov.), red spider mite (Tetranychus cinnabarinus Boisd.), grey weevil (Myllocerus viridanus Fab.), cotton whitefly (Bemesia tabaci Genn.), spiralling whitefly (Aleurodicus dispersus Russel) and scale (Aspidiotus destructor Sign.) (Regupathy et al., 2003; Pena et al., 2005). Recently, it was observed that tapioca was affected severely by mealybug Paracoccus marginatus (Williams and Granara de Willink) (Pseudococcidae: Hemiptera) (Muniappan,

2009; Suresh et al., 2010). Papaya mealybug is a polyphagous pest that can damage a large number of economically important field crops, tropical and sub-tropical fruits, vegetables and ornamental plants. It has caused an estimated loss of about Rs. 300 crores in each state (Anonymous, 2010). It became serious pest of papaya in Tamil Nadu and nearly 820 ha in Erode and 576 ha in Coimbatore had been affected (Revathy, 2010). Entire papain industry at Coimbatore was lost due to the severity of the pest on papaya, which spread to tapioca and mulberry in neighbouring districts of Tamil Nadu (Suresh et al., 2010). 
The papaya mealybug is believed to be the native of Mexico or Central America and was first described in 1992 by Williams and Granara de Willink and re-described in 2002 by Miller and Miller Papaya mealybug infestations are typically observed as clusters of cotton-like masses on the above-ground portion of plants. Colonization of mealybugs on tapioca has been noted along the veins and the midribs of the older leaves and all areas of tender leaves (Walker et al., 2006). Severely affected older leaves turn yellow and dry up. Tender leaves become bunched and distorted. Heavy mealybug populations produce a large volume of honey dew, which causes black sooty mould on the infested fruits and vegetation (Meyerdirk et al., 2004).

\section{Materials and Methods}

\section{Survey for $P$. marginatus incidence in papaya field}

One time survey was undertaken during 2011 to 2012 in 95 farmers' holdings in Coimbatore, Erode, Perambalur, Salem and Tripur districts of Tamil Nadu. Five spots were selected in each field and in each spot 20 plants were selected at random (totally 100 plants / field) and observed for mealybug incidence.

The per cent incidence of mealybug infested plant was worked out from the number of plants affected and total number of plants observed by following the formula,

Per cent Incidence $=$

$$
\text { Number of plants observed }
$$

Infestation of the mealybug was categorized on the following parameters based on visual observations.

\section{Results and Discussion}

Survey was made during year 2011 to 2012 in different blocks of Coimbatore, Erode, Perambalur, Salem and Tirupur districts of Tamil Nadu on the incidence of papaya mealybug, P. marginatus on tapioca. Among the ninety five farmer's holdings observed, none of the field was free from the pest incidence. The incidence ranged from 2 to 100 per cent.

All the plant parts were covered with mealybugs with crinkling of leaves and leaves and fruits covered with honey dew and sooty mold and were started drying. Among the 95 fields surveyed, 13 recorded very high incidence, 3 with high incidence, 5 with medium incidence, 22 with low incidence and 52 with very low incidence. Very high incidence of 100 per cent was observed in only eight fields and data presented in tables 2, 3, 4, and 5. Complete shedding of leaves occurred in most of the plants.

Surveys conducted in tapioca fields at different blocks of Coimbatore, Erode, Perambalur, Salem and Tirupur districts of Tamil Nadu revealed that the incidence of papaya mealybug ranged from 2 to 100 per cent in tapioca in 95 farmers' holding fields'. Earlier observations by Regupathy and Ayyasamy (2010) in 81 holdings across Tamil Nadu revealed 1 to 100 per cent incidence in tapioca. $P$. marginatus was recorded in papaya for the first time in Tamil Nadu Agricultural University, Coimbatore, during July, 2008 by Muniappan (2009) and was also observed on commercial papaya plantations in Udumalpet, Karur, Coimbatore and Erode as well as on mulberry and jatropha fields in 11 locations in and around Coimbatore (Table 1).

The level of incidence of $P$. marginatus varied from 0 to 60 per cent in Coimbatore, Erode and Tirupur districts (Suresh et al., 2010). 
Surveys conducted during May 2009 between latitude and longitude ranges of $10^{\circ} .56^{\prime}-11^{\circ}$.

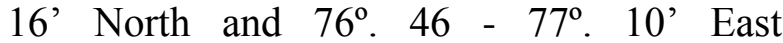
respectively in Coimbatore district of Tamil Nadu registered heavy infestations of $P$. marginatus in plantation of papaya (Carica papaya L.: Caricaceae), mulberry (Morus alba L.: Moraceae), jatropha (Jatropha curcus L.: Euphorbiaceae) and tapioca (Manihot esculenta C.: Euphorbiaceae) besides moderate to low infestations on the shoe flower (Hibiscus- rosa- sinensis L.: Malvaceae), guava (Psidium guajava L.: Myrtaceae), brinjal (Solanum melongena L.: Solanaceae) and tomato (Lycopersicon esculentum L.: Solanaceae) (Anonymous, 2009).

Table.1 Grade chart for estimating the pest intensity level of $P$. marginatus in tapioca as adopted by Regupathy and Ayyasamy, 2010

\begin{tabular}{ll}
\hline \multicolumn{1}{c}{ Grade } & \multicolumn{1}{c}{ Infestation levels } \\
\hline Very Low & i. Few individuals of the mealybug casually found \\
i. Mealybug found in low numbers \\
ii. No adverse symptoms like deformation of leaf observed on the plant \\
Medium & i. Almost 75 to 100 per cent coverage of leaves / fruits/ inflorescence \\
& ii. Yellowing of leaves \\
& iii. Shedding of infested leaves and fruits \\
\hline Grade & i. Almost all plant parts (stem, leaves, flowers and fruits) covered with \\
& mealybug showing white appearance \\
& ii. Leaves, fruits and inflorescences covered with honey dew excretion and \\
& sooty mould \\
i. All plant parts (stem, leaves, flowers and fruits) covered with mealybug \\
showing white appearance
\end{tabular}


Table.2 Infestation levels of $P$. marginatus on tapioca at different locations in Coimbatore District

\begin{tabular}{|c|c|c|c|c|c|c|}
\hline $\begin{array}{l}\text { S. } \\
\text { No }\end{array}$ & Location & $\begin{array}{c}\text { Area } \\
\text { (Acre) }\end{array}$ & $\begin{array}{c}\text { Age } \\
\text { (Months) }\end{array}$ & $\begin{array}{l}\text { Variety/ } \\
\text { Hybrid }\end{array}$ & $\begin{array}{c}\text { Pest } \\
\text { Incidence } \\
(\%) \\
\end{array}$ & Pest Intensity \\
\hline 1 & $\begin{array}{l}\text { Kinathukadavu } \\
\text { Coimbatore }\end{array}$ & 2.00 & 8 & Mulvadi & 100 & Very high \\
\hline 2 & $\begin{array}{l}\text { Kinathukadavu } \\
\text { Coimbatore }\end{array}$ & 1.50 & 7 & Mulvadi & 94 & Very high \\
\hline 3 & $\begin{array}{l}\text { Kinathukadavu } \\
\text { Coimbatore }\end{array}$ & 1.50 & 7 & Mulvadi & 84 & Very high \\
\hline 4 & $\begin{array}{l}\text { Kalankattuputhur } \\
\text { Coimbatore }\end{array}$ & 2.00 & 7 & Mulvadi & 78 & High \\
\hline 5 & Athurpollachi Pollachi & 5.00 & 10 & Mulvadi & 56 & Medium \\
\hline 6 & Athurpollachi Pollachi & 4.00 & 5 & Mulvadi & 45 & Medium \\
\hline 7 & Athurpollachi Pollachi & 1.00 & 2 & White rose & 30 & Low \\
\hline 8 & Athurpollachi Pollachi & 1.00 & 10 & Mulvadi & 22 & Low \\
\hline 9 & Athurpollachi Pollachi & 1.00 & 10 & Mulvadi & 26 & Low \\
\hline 10 & Vadakkipalaiyam Pollachi & 2.00 & 12 & Mulvadi & 33 & Low \\
\hline 11 & Vadakkipalaiyam Pollachi & 1.00 & 12 & Whiterose & 37 & Low \\
\hline 12 & Vadakkipalaiyam Pollachi & 2.00 & 12 & Whiterose & 26 & Low \\
\hline 13 & Vadakkipalaiyam Pollachi & 1.00 & 12 & Whiterose & 29 & Low \\
\hline 14 & Nalloore Pollachi & 1.50 & 11 & Mulvadi & 39 & Low \\
\hline 15 & Nalloore Pollachi & 1.00 & 10 & White rose & 22 & Low \\
\hline 16 & Nalloore Pollachi & 1.50 & 11 & Mulvadi & 20 & Low \\
\hline 17 & Nalloore Pollachi & 2.00 & 10 & Mulvadi & 92 & Very high \\
\hline 18 & $\begin{array}{l}\text { Narasipuramroad } \\
\text { Thondamuthur }\end{array}$ & 2.50 & 6 & Mulvadi & 100 & Very high \\
\hline 19 & $\begin{array}{l}\text { Narasipuramroad } \\
\text { Thondamuthur }\end{array}$ & 1.00 & 6 & Mulvadi & 92 & Very high \\
\hline 20 & $\begin{array}{l}\text { Narasipuramroad } \\
\text { Thondamuthur }\end{array}$ & 3.00 & 5 & Mulvadi & 78 & High \\
\hline 21 & $\begin{array}{l}\text { Mathampalayam } \\
\text { Periyanayakkan } \\
\text { palayam }\end{array}$ & 1.00 & 7 & Mulvadi & 100 & Very high \\
\hline 22 & $\begin{array}{l}\text { Mathampalayam } \\
\text { Periyanayakkan palayam }\end{array}$ & 1.00 & 5 & Mulvadi & 45 & Medium \\
\hline 23 & Bellathi Karamadai & 1.00 & 8 & Mulvadi & 100 & Very high \\
\hline 24 & $\begin{array}{l}\text { Onnipalayapudhur } \\
\text { Periyanayakkanpalayam }\end{array}$ & 1.50 & 3 & Mulvadi & 8 & Very low \\
\hline 25 & $\begin{array}{l}\text { Onnipalayapudhur } \\
\text { Periyanayakkanpalayam }\end{array}$ & 1.50 & 4 & Mulvadi & 6 & Very low \\
\hline 26 & $\begin{array}{l}\text { Onnipalayapudhur } \\
\text { Periyanayakkanpalayam }\end{array}$ & 3.00 & 9 & Mulvadi & 100 & Very high \\
\hline 27 & $\begin{array}{l}\text { Onnipalayapudhur } \\
\text { Periyanayakkanpalayam }\end{array}$ & 1.50 & 5 & Mulvadi & 90 & Very high \\
\hline 28 & $\begin{array}{l}\text { Onnipalayapudhur } \\
\text { Periyanayakkanpalayam }\end{array}$ & 1.00 & 5 & Mulvadi & 65 & High \\
\hline 29 & Vellamadai Kovilpalayam & 1.50 & 8 & Mulvadi & 22 & Low \\
\hline 30 & Vellamadai Kovilpalayam & 0.50 & 9 & Mulvadi & 10 & Very low \\
\hline 31 & $\begin{array}{l}\text { Devampalaiyam } \\
\text { Kovilpalayam }\end{array}$ & 0.50 & 5 & Mulvadi & 17 & Low \\
\hline
\end{tabular}


Table.3 Infestation levels of P. marginatus on tapioca at Annur block of Coimbatore district

\begin{tabular}{|c|c|c|c|c|c|c|}
\hline $\begin{array}{l}\text { S. } \\
\text { No }\end{array}$ & Location & $\begin{array}{c}\text { Area } \\
\text { (Acre) }\end{array}$ & $\begin{array}{c}\text { Age } \\
\text { (Months) }\end{array}$ & Variety/Hybrid & $\begin{array}{c}\text { Pest } \\
\text { Incidence } \\
(\%)\end{array}$ & $\begin{array}{c}\text { Pest } \\
\text { Intensity }\end{array}$ \\
\hline 1 & Pasur Annur & 1.75 & 9 & Mulvadi & 16 & Low \\
\hline 2 & Pasur Annur & 1.75 & 6 & Mulvadi & 9 & Very low \\
\hline 3 & Pasur Annur & 1.00 & 6 & Mulvadi & 6 & Very low \\
\hline 4 & $\begin{array}{l}\text { Pongalore } \\
\text { Annur }\end{array}$ & 2.50 & 7 & Mulvadi & 10 & Very low \\
\hline 5 & $\begin{array}{l}\text { Pongalore } \\
\text { Annur }\end{array}$ & 2.50 & 10 & Mulvadi & 8 & Very low \\
\hline 6 & $\begin{array}{l}\text { Sokkampalayam } \\
\text { Annur }\end{array}$ & 3.00 & 10 & Mulvadi & 13 & Low \\
\hline 7 & $\begin{array}{l}\text { Thokkupalayam } \\
\text { Annur }\end{array}$ & 1.00 & 2 & Mulvadi & 6 & Very low \\
\hline 8 & $\begin{array}{l}\text { Thokkupalayam } \\
\text { Annur }\end{array}$ & 3.00 & 2 & Mulvadi & 8 & Very low \\
\hline 9 & Pasur Annur & 1.50 & 8 & Mulvadi & 5 & Very low \\
\hline 10 & Pasur Annur & 1.50 & 7 & White rose & 8 & Very low \\
\hline 11 & Pasur Annur & 0.50 & 3 & White rose & 7 & Very low \\
\hline 12 & Pasur Annur & 1.50 & 8 & White rose & 10 & Very low \\
\hline 13 & Pasur Annur & 1.50 & 7 & Mulvadi & 5 & Very low \\
\hline 14 & Pasur Annur & 0.50 & 3 & Mulvadi & 2 & Very low \\
\hline 15 & Pasur Annur & 2.50 & 5 & Mulvadi & 7 & Very low \\
\hline 16 & Pasur Annur & 1.00 & 5 & Mulvadi & 3 & Very low \\
\hline 17 & Pasur Annur & 1.00 & 4 & Mulvadi & 6 & Very low \\
\hline 18 & Pasur Annur & 1.00 & 2 & Mulvadi & 9 & Very low \\
\hline 19 & Pasur Annur & 2.00 & 2 & Mulvadi & 5 & Very low \\
\hline 20 & Pasur Annur & 1.00 & 2 & Mulvadi & 8 & Very low \\
\hline 21 & Pasur Annur & 1.50 & 2 & Mulvadi & 7 & Very low \\
\hline 22 & Pasur Annur & 1.50 & 2 & Mulvadi & 7 & Very low \\
\hline 23 & Pasur Annur & 1.50 & 2 & Mulvadi & 9 & Very low \\
\hline 24 & Pasur Annur & 1.00 & 2 & Mulvadi & 6 & Very low \\
\hline 25 & $\begin{array}{l}\text { Allapalayam } \\
\text { Annur }\end{array}$ & 0.50 & 9 & Mulvadi & 5 & Very low \\
\hline 26 & Pasur Annur & 4.00 & 4 & Mulvadi & 10 & Very low \\
\hline 27 & Pasur Annur & 0.75 & 8 & Mulvadi & 10 & Very low \\
\hline 28 & $\begin{array}{l}\text { Allapalayam } \\
\text { Annur }\end{array}$ & 1.00 & 7 & Mulvadi & 7 & Very low \\
\hline
\end{tabular}


Table.4 Infestation levels of $P$. marginatus on tapioca at different locations in Perambalur District

\begin{tabular}{|c|c|c|c|c|c|c|}
\hline S. No & Location & $\begin{array}{c}\text { Area } \\
\text { (Acre) }\end{array}$ & $\begin{array}{c}\text { Age } \\
\text { (Months) }\end{array}$ & Variety/Hybrid & $\begin{array}{c}\text { Pest } \\
\text { incidence } \\
(\%)\end{array}$ & $\begin{array}{c}\text { Pest } \\
\text { intensity }\end{array}$ \\
\hline 1 & $\begin{array}{l}\text { Sengunam } \\
\text { Perambalur }\end{array}$ & 1.00 & 10 & Mulvadi & 7 & Very low \\
\hline 2 & $\begin{array}{l}\text { Sengunam } \\
\text { Perambalur }\end{array}$ & 1.50 & 11 & Mulvadi & 5 & Very low \\
\hline 3 & $\begin{array}{l}\text { Somanapudhur } \\
\text { Perambalur }\end{array}$ & 1.00 & 10 & Mulvadi & 5 & Very low \\
\hline 4 & $\begin{array}{l}\text { Sengunam } \\
\text { Perambalur }\end{array}$ & 2.00 & 11 & Mulvadi & 10 & Very low \\
\hline 5 & $\begin{array}{l}\text { Sengunam } \\
\text { Perambalur }\end{array}$ & 2.00 & 11 & Mulvadi & 14 & Very low \\
\hline 6 & $\begin{array}{l}\text { Sengunam } \\
\text { Perambalur }\end{array}$ & 1.50 & 11 & Mulvadi & 6 & Very low \\
\hline 7 & $\begin{array}{l}\text { Poombukar } \\
\text { Veppanthattai }\end{array}$ & 1.00 & 12 & Mulvadi & 5 & Very low \\
\hline 8 & $\begin{array}{l}\text { Poombukar } \\
\text { Veppanthattai }\end{array}$ & 1.00 & 11 & Mulvadi & 8 & Very low \\
\hline 9 & $\begin{array}{l}\text { Annamangalam, } \\
\text { Veppanthattai }\end{array}$ & 3.00 & 11 & Mulvadi & 10 & Very low \\
\hline 10 & $\begin{array}{l}\text { Annamangalam, } \\
\text { Veppanthattai }\end{array}$ & 4.00 & 11 & Mulvadi & 7 & Very low \\
\hline 11 & $\begin{array}{l}\text { Poombukar } \\
\text { Veppanthattai }\end{array}$ & 3.00 & 11 & Mulvadi & 6 & Very low \\
\hline 12 & $\begin{array}{l}\text { P.R.Nallor } \\
\text { Alathur }\end{array}$ & 6.00 & 10 & Mulvadi & 9 & Very low \\
\hline 13 & $\begin{array}{l}\text { Jaminparaiyur } \\
\text { Alathur }\end{array}$ & 2.00 & 10 & Mulvadi & 5 & Very low \\
\hline 14 & $\begin{array}{l}\text { P.R.Nallor } \\
\text { Alathur }\end{array}$ & 3.00 & 12 & Mulvadi & 9 & Very low \\
\hline 15 & $\begin{array}{l}\text { P.R.Nallor } \\
\text { Alathur }\end{array}$ & 2.00 & 10 & Mulvadi & 10 & Very low \\
\hline 16 & $\begin{array}{l}\text { P.R.Nallor } \\
\text { Alathur }\end{array}$ & 6.00 & 10 & Mulvadi & 5 & Very low \\
\hline 17 & $\begin{array}{l}\text { K.Pudhur } \\
\text { Veppur }\end{array}$ & 3.50 & 12 & Mulvadi & 7 & Very low \\
\hline 18 & $\begin{array}{l}\text { Namiyur } \\
\text { Veppur }\end{array}$ & 1.50 & 10 & Mulvadi & 9 & Very low \\
\hline 19 & $\begin{array}{l}\text { Namiyur } \\
\text { Veppur }\end{array}$ & 1.50 & 10 & Mulvadi & 4 & Very low \\
\hline 20 & $\begin{array}{l}\text { K.Pudhur } \\
\text { Veppur }\end{array}$ & 1.00 & 10 & Mulvadi & 4 & Very low \\
\hline 21 & $\begin{array}{l}\text { K.Pudhur } \\
\text { Veppur }\end{array}$ & 3.00 & 11 & Mulvadi & 9 & Very low \\
\hline 22 & $\begin{array}{l}\text { K.Pudhur } \\
\text { Veppur }\end{array}$ & 2.00 & 11 & Mulvadi & 8 & Very low \\
\hline 23 & $\begin{array}{l}\text { K.Pudhur } \\
\text { Veppur }\end{array}$ & 1.50 & 11 & Mulvadi & 6 & Very low \\
\hline
\end{tabular}


Table.5 Infestation levels of $P$. marginatus on tapioca at different locations in Salem and Tirupur Districts

\begin{tabular}{|c|c|c|c|c|c|c|}
\hline $\begin{array}{l}\text { S. } \\
\text { No }\end{array}$ & Location & $\begin{array}{c}\text { Area } \\
\text { (Acre) }\end{array}$ & $\begin{array}{c}\text { Age } \\
\text { (Months) }\end{array}$ & Variety/Hybrid & $\begin{array}{c}\text { Pest } \\
\text { Incidence } \\
(\%)\end{array}$ & $\begin{array}{c}\text { Pest } \\
\text { Intensity }\end{array}$ \\
\hline 1 & Karumandurai, Karumandurai & 4.00 & 8 & Mulvadi & 100 & $\begin{array}{l}\text { Very } \\
\text { high }\end{array}$ \\
\hline 2 & Karumandurai, Karumandurai & 5.00 & 7 & Mulvadi & 100 & $\begin{array}{l}\text { Very } \\
\text { high }\end{array}$ \\
\hline 3 & Karumandurai, Karumandurai & 4.00 & 8 & Mulvadi & 100 & $\begin{array}{l}\text { Very } \\
\text { high }\end{array}$ \\
\hline 4 & Peranayakkan pudhur, Avinasi & 4.00 & 7 & Mulvadi & 55 & Medium \\
\hline 5 & Peranayakkan pudhur Avinasi & 3.00 & 7 & Mulvadi & 44 & Medium \\
\hline 6 & Peranayakkan pudhur Avinasi & 2.00 & 5 & Mulvadi & 26 & Low \\
\hline 7 & Neelipalayam Avinasi & 1.00 & 5 & Mulvadi & 55 & Low \\
\hline 8 & Peranayakkan pudhur Avinasi & 2.00 & 5 & Mulvadi & 37 & Low \\
\hline 9 & Neelipalayam Avinasi & 1.00 & 5 & Mulvadi & 28 & Low \\
\hline 10 & Neelipalayam Avinasi & 2.00 & 2 & Mulvadi & 22 & Low \\
\hline 11 & Neelipalayam Avinasi & 1.00 & 9 & Mulvadi & 27 & Low \\
\hline 12 & Nochikottai Avinasi & 1.00 & 10 & Mulvadi & 33 & Low \\
\hline 13 & Nochikottai Avinasi & 1.00 & 10 & Mulvadi & 39 & Low \\
\hline
\end{tabular}

Papaya mealybug has a wide host range sparring more than 22 plant families (BenDov, 2008) and more than 50 hosts (Regupathy and Ayyasamy, 2010), which includes field and horticultural crops, herbaceous perennials, climbers, trees, ornamentals and several weed hosts.

In conclusion, investigations were made on the incidence level of papaya mealybug, Paracoccus marginatus (Williams and Granara de Willink). A total of 95 tapioca farmers' fields were surveyed for infestation by $P$. marginatus in Coimbatore, Erod, Perambalur, Salem and Tirupur districts of Tamil Nadu. The incidence of $P$. marginatus intensity ranged from very low to very high (2 to 100 per cent) in tapioca.

\section{Acknowledgement}

Author very much thankfull to Dr.S.Suresh for his svaluable guidance in identification of papaya mealybug.

\section{References}

Anonymous. 2009. Pest alert on the spread of mealybugs in Tamil Nadu.

Anonymous. 2010. Parasitoids for biological control of papaya mealybug. The Hindu, October 8, Coimbatore.

Ben-Dov, Y. 2008. Scale Net, Paracoccus marginatus. 17 September 2008.

Meyerdirk, D. E., R. Muniappan, R. Warkentin, J. Bamba and G. V. P. Reddy. 2004. Biological control of the papaya mealybug, Paracoccus marginatus (Hemiptera: Pseudococcidae) in Guam. Plant Prot. Qrtly., 19: 110-114

Miller, D. R. and G. L. Mliller. 2002. Redescription of Paracoccus marginatus Williams and Granara de Willink (Hemiptera: Coccoidea: Pseudococcidae), including descriptions of the immature stages and adult male. In: Proc. Entomol. Soc. Washington., 104: 1-23.

Muniappan, R. 2009. Papaya mealybug, a new invader in Asia. IAPPS Newsletter November1. January, 2009.

Pena, J. E., A. Pantoja, L. Osborne, R. Duncan, M. D. Coss-Flores, C. Meister, S. Halbert, 
G. Evans and A. Hammon. 2005. Homopteran and mite pests of papaya and their control. Proc. Fla. State Hort. Soc., 118: 221-227.

Regupathy, A., S. Palanisamy, N. Chandramohan and K. Gunathilagaraj. 2003. Guide on Crop Pests, Fourth Edition. Sheeba Printers, Coimbatore, 276p

Regupathy, A. and R. Ayyasamy. 2010. Infestation of the invasive papaya mealybug Paracoccus marginatus (Williams and Granara de Willink) in small scale papaya farming system in Tamil Nadu. Hexapoda, 17(1): 12-20.

Revathy, R. 2010. Chemical control of papaya mealybug, Paracoccus marginatus
(Williams and Granara de Willink) on papaya. M.Sc. (Agri) Thesis. Tamil Nadu Agric. Univ., Coimbatore, India. 125p

Suresh, S., R. Jothimani, P. Sivasubrmanian, P. Karuppuchamy, R. Samiyappan and E. I. Jonathan. 2010. Invasive mealybugs of Tamil Nadu and their management. Karnataka J. Agric. Sci., 23(1): 26-34.

Walker, A., M. Hoy and D. Meyerdirk. 2006. Papaya mealybug (Paracoccus marginatus Williams and Granara de Willink (Insecta: Hemiptera: Pseudococcidae). EENY-302. Featured Creatures. Entomology and Nematology Department, Florida Cooperative Extension Service, Institute

\section{How to cite this article:}

Indirakumar, K., J.S. Kennedy and Devi, M. 2017. Survey of Papaya Mealybug Paracoccus marginatus on Tapioca Crop in Different Districts of Tamil Nadu. Int.J.Curr.Microbiol.App.Sci. 6(2): 503-510. doi: http://dx.doi.org/10.20546/ijcmas.2017.602.057 